\title{
Changes in the incidence and prevalence of type 1 and type 2 diabetes among 2 million children and adolescents in Hungary between 2001 and 2016 - a nationwide population-based study
}

\author{
László Barkai ${ }^{1,2}$, Zoltán Kiss³ , György Rokszin ${ }^{4}$, Zsolt Abonyi-Tóth ${ }^{5}$, György Jermendy ${ }^{6}$, \\ István Wittmann ${ }^{3}$, Péter Kempler ${ }^{7}$
}

\author{
${ }^{1}$ Institute of Theoretical Health Sciences, Faculty of Health Care, University of Miskolc, \\ Miskolc, Hungary \\ ${ }^{2}$ Department of Paediatrics and Adolescent Medicine, Faculty of Medicine, Pavol Jozef \\ Safarik University, Kosice, Slovakia \\ ${ }^{3} 2^{\text {nd }}$ Department of Medicine and Nephrological Center, Medical Faculty, University \\ of Pécs, Pécs, Hungary \\ ${ }^{4}$ RxTarget Statistical Agency, Szolnok, Hungary \\ ${ }^{5}$ University of Veterinary Medicine, Budapest, Hungary \\ ${ }^{6}$ Bajcsy-Zsilinszky Hospital, Budapest, Hungary \\ ${ }^{7} 1^{\text {st }}$ Department of Medicine, Faculty of Medicine, Semmelweis University, Budapest, \\ Hungary
}

Submitted: 7 April 2019

Accepted: 13 August 2019

Arch Med Sci 2020; 16 (1): 34-41

DOI: https://doi.org/10.5114/aoms.2019.88406

Copyright (c) 2019 Termedia \& Banach

\section{Abstract}

Introduction: The aim of the present study was to assess changes in the incidence and prevalence of type 1 diabetes (T1DM) and type 2 diabetes (T2DM) in children and adolescents in Hungary during the period 2001 to 2016 in order to provide nationwide population-based epidemiology data on diabetes in youths aged $0-18$ years.

Material and methods: This was a retrospective cohort study of Hungarian children and adolescents aged 18 years or younger. Pharmacologically treated diabetes cases were obtained through a population-based registry of the Hungarian National Health Insurance Fund. Time series analysis was used to evaluate the changing patterns of the incidence and prevalence for type 1 and type 2 diabetes covering a 16 -year period.

Results: During the study period, 6,138 and 1,997 new T1DM and T2DM cases were observed, respectively. Newly diagnosed T2DM cases accounted for $24.5 \%$ of all incident diabetes cases. Incidence of T1DM increased from $16 / 100,000$ to $23 / 100,000\left(R^{2}=0.7681 ; p<0.0001\right)$. The male-to-female ratio among newly diagnosed T1DM patients did not change over the study period. Prevalence of T1DM rose from $114 / 100,000$ to $209 / 100,000\left(R^{2}=\right.$ $0.9909 ; p<0.0001)$. The prevalent T1DM cases showed significant male predominance in every year $(p<0.05)$. Incidence of T2DM decreased from $8 / 100,000$ to $5 / 100,000\left(R^{2}=0.4977 ; p<0.0014\right)$. The overall prevalence of T2DM did not change significantly. Prevalent T2DM cases showed significant female predominance in every year $(p<0.0001)$. A significant decrease in male-to female ratio was observed among newly diagnosed T2DM cases over the study period $(p<0.0001)$.

Conclusions: According to these population-based Hungarian data of children and adolescents with diabetes, T1DM is still the most common form and its frequency continues to rise, affecting more males than females. A high proportion of patients have T2DM, affecting more females than

\section{Corresponding author:}

László Barkai MD

Egyetemváros

3515 Miskolc, Hungary

Phone: +36 309384467

E-mail: barkai.l@t-online.hu 
males, but the occurrence of medically treated cases is not increasing. The decrease in male-to-female ratio in newly diagnosed T2DM cases needs further investigations.

Key words: type 1 diabetes, type 2 diabetes, incidence, prevalence, children, adolescents.

\section{Introduction}

Diabetes, mainly type 2 , in adult populations represents a public health and economic burden all over the world and its frequency is increasing in almost every country [1, 2]. Diabetes is one of the most common chronic conditions of childhood and disease management and risks of acute and chronic complications pose significant challenges to the health care system [3, 4]. Incidence and prevalence data of diabetes in children and adolescents also have shown an increase during the past decades, although tendencies for type 1 and type 2 diabetes are different.

The vast majority of cases of diabetes in childhood are type 1 diabetes (T1DM), accounting for over $90 \%$ of all diabetes cases, and its incidence varies greatly between different countries, within countries, and between different ethnic populations [5]. Worldwide, the incidence of T1DM diabetes is increasing by approximately $3-5 \%$ per annum [6-10]. However, the rates of increase and temporal trends are variable, and recently, a slowing rise in incidence has been reported in some countries [11-13].

Increasing incidence and prevalence of type 2 diabetes (T2DM) have been observed since the early 1990s, particularly in the US, accounting for a significant proportion of youth onset diabetes in certain at-risk populations [14-17]. European countries and Australia reported lower incidence than the US [18-21], but an increase has been observed in the UK [22-24].

Several population-based epidemiological data are available regarding T1DM, but more limited observations have been reported about T2DM in children and adolescents. Most of the studies documented incidence for T1DM [6-13] and prevalence for T2DM [14, 17-20]; some of them report both incidence and prevalence for T1DM [23] or T2DM [24]. However, a parallel report of incidence and prevalence data for both T1DM and T2DM in youths within the same population is lacking.

Representative epidemiology data are important from a health policy point of view as it will enable the monitoring of disease burden, which is directly related to the planning of health care resources [25, 26]. Having nationwide population-based reliable and exact data on the occurrence of diabetes in youths may support clinicians and policy makers to predict health and economic impacts of this condition and its long-term complications.

The purpose of the present study was to assess the incidence and prevalence of both T1DM and T2DM in children and adolescents in Hungary during the period 2001 to 2016 in order to provide nationwide population-based accurate figures for diabetes in youths aged 0-18 years.

\section{Material and methods}

This was a retrospective cohort study covering a 16-year period from 2001 to 2016 . The study forms part of the Hungarian Diabetes Association's initiative to analyze diabetes patients' data extracted from the Hungarian National Health Insurance Fund (HNHIF). This database contains patient records of the whole population of the country. Information held in the database includes patient demographics, medical diagnosis coded as the International Classification of Diseases (ICD) and prescriptions details coded according to the Anatomical Therapeutic Chemical (ATC) classification. The database of the Hungarian National Health Insurance Fund does not record laboratory results such as glycated hemoglobin $\left(\mathrm{HbA}_{1 \mathrm{c}}\right.$ ) or cholesterol level or any anthropometric parameters of diabetes patients such as body mass index (BMI) or body weight, and therefore these data were not available for analysis.

Patients were included in our study if they had at least 2 prescriptions of antidiabetic treatment (ATC A10) between 1 January, 2001 and 31 December, 2016 or one prescription of antidiabetic treatment together with at least one occurrence of diabetes related ICD code (E10-E14). We excluded those patients who had a diagnosis of polycystic ovary syndrome (ICD 10 E282), gestational diabetes (10 024) or if they exceeded age 18 in the given year. If a patient had only insulin therapies or had a maximum of 3 oral antidiabetic prescriptions in the first half year together with insulin treatment, we counted the patient in the T1DM population [27]. Those patients who were included but not matching T1DM were considered as patients with T2DM. Date of diabetes diagnosis was defined as the first occurrences of the diabetes ICD code (E10-E14) in the database for a patient or the first antidiabetic treatment, whichever occurred earlier. With these aspects, we could clarify the incidence and prevalence of 0-18-year-old diabetic population. Incidence of diabetes was de- 
fined for patients who did not match the inclusion criteria in prior years including the screening period. If patients were alive on the $1^{\text {st }}$ of January and matched all inclusion criteria, we counted them in the prevalent population of the given year regardless of whether they were diagnosed later in the same year or identified with diabetes in prior years. We had a limitation of the HNHIF database as only aggregated data for the 0-18-year-old cohort was available. Therefore, we were not able to obtain age-standardized data in more details.

Children and adolescents aged 18 years or younger on the $1^{\text {st }}$ of January were selected for every single year in the study period. Incidences and prevalences were calculated as the number of cases per 100,000 per calendar year. If a patient's age exceeded 18 years, regardless of being included in prevalence and incidence calculations in prior years, the case was extracted from the analysis. The $R$ Project for Statistical Computing, R version 3.4.2 was used for statistical analysis (R Core Team 2017, Vienna, Austria, URL https://www.R-project.org/) to assess regression, mean annual change in incidence and prevalence with $95 \%$ confidence intervals. The math algorithm needed for the research was prepared by the RxTarget Statistical Programming and Analysis Company based on the preliminary research plan, using sql and plsql program language, which was sent to HNHIF by e-mail. The algorithm was run by the HNHIF specialists using ID code based data of the patients who had been dissected. The Fund Manager has issued the completed data tables on a separate license (HNHIF license number: S04/161/2016), anonymously, free of charge, in aggregate form, with all unique identifiers, with the utmost consideration of data protection rules. Change in male-to-female ratio was calculated with binominal regression analysis.

\section{Results}

\section{Nationwide reference population}

The reference population included Hungarian youths aged 0-18 years between 2001 and 2016, inclusive. A gradual and constant decrease in the total population of youth could be observed affecting both genders similarly (male/female ratio: 1.05). During the period investigated, the reference population decreased significantly by $18.0 \%$ $\left(R^{2}=0.9929 ; p<0.0001\right)$ from $2,217,202$ in 2001 to $1,818,413$ in 2016 and the mean annual rate of the change was approximately $-1.2 \% /$ year $(95 \% \mathrm{Cl}$ : $-1.3--1.1$ )

\section{Type 1 diabetes}

Incident and prevalent cases of T1DM are shown in Table I, and incidence and prevalence rates are depicted in Figures 1 and 2, respectively.
Altogether, 6,138 new T1DM cases $(3,407$ males and 2,731 females) were observed between 2001 and 2016, inclusive (male/female ratio: 1.25). No significant change in the male-to-female ratio of incident cases was noted over the study period. Incidence of T1DM increased significantly and almost continuously from 16/100,000 at the beginning, reaching the highest annual rate of $23 / 100,000$ by the end of the 16-year period investigated $\left(R^{2}=0.7681 ; p<0.0001\right)$. The mean annual rate of the increase in the incidence of T1DM was $2.5 \%$ /year (95\% Cl: $1.8-3.2)$ during the study period.

Prevalence of T1DM rose constantly from $114 / 100,000$ to $209 / 100,000$ during the study period $\left(R^{2}=0.9909 ; p<0.0001\right)$. The mean annual rate of the increase in the prevalence of T1DM was $5.8 \%$ /year $(95 \% \mathrm{Cl}: 5.5-6.1)$ during the assessments. Prevalent cases showed significant male predominance in every year $(p<0.05)$.

\section{Type 2 diabetes}

Incident and prevalent cases of T2DM are shown in Table I, and incidence and prevalence rates are depicted in Figures 3 and 4, respectively.

Altogether, 1,997 new T2DM cases (516 males and 1.481 females) were observed between 2001 and 2016, inclusive (female/male ratio: 2.86 ), accounting for $24.5 \%$ of all incident diabetes cases. Male-to-female ratio of incident cases decreased significantly over the study period $(p<0.0001)$. New T2DM cases accounted for $34.5 \%$ and $18.1 \%$ of all newly diagnosed diabetes cases in 2001 and 2016, respectively. Incidence rate of T2DM showed some fluctuation (between 8/100,000 in 2001 and 5/100,000 in 2016), but a clear and significant decreasing trend was seen during the study period, reaching the lower rates by the end of the period investigated $\left(R^{2}=0.4977 ; p<0.0014\right)$. The mean annual rate of the change in the incidence of T2DM was $-2.5 \%$ /year $(95 \% \mathrm{Cl}:-3.8--1.3)$ during the study period.

Prevalence of T2DM rose between 2001 and 2006 from 20/100,000 to $36 / 100,000$ and decreased thereafter to $22 / 100,000$ by the end of the period investigated. Prevalent T2DM cases accounted for $14.6 \%$ and $9.4 \%$ of all prevalent diabetes cases in 2001 and 2016, respectively. The overall change of prevalence was not significant $\left(R^{2}=-0.0009 ; p=0.3375\right)$ over the 16-year period. Prevalent cases showed significant female predominance in every year $(p<0.0001)$.

\section{Discussion}

In the current population-based 16-year study, constant increases in the incidence and prevalence of T1DM were documented among children and 


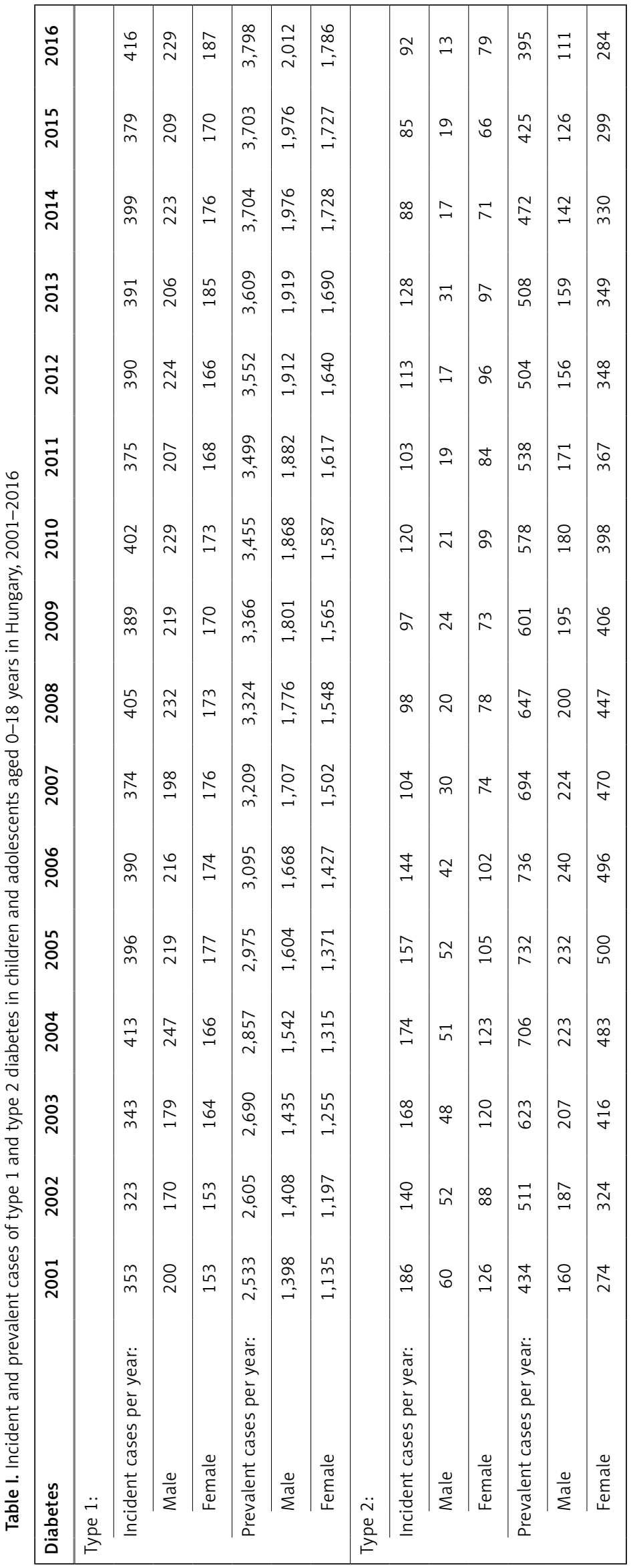




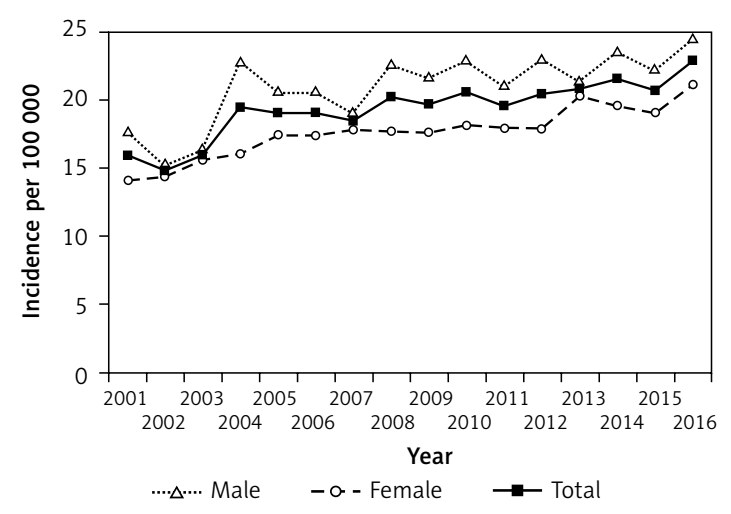

Figure 1. Incidence of type 1 diabetes in children and adolescents aged 0-18 years in Hungary between 2001 and 2016, inclusive

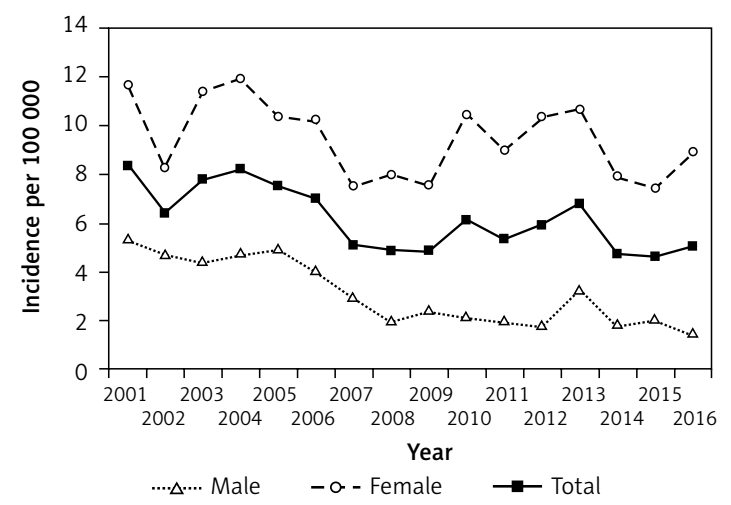

Figure 3. Incidence of type 2 diabetes in children and adolescents aged $0-18$ years in Hungary between 2001 and 2016, inclusive

adolescents. In contrast, a decreasing incidence of T2DM was observed in the same population. The annual rate of increase in T1DM incidence was $2.5 \% /$ year and the annual rate of change in $\mathrm{T} 2 \mathrm{DM}$ incidence was $-2.5 \% /$ year. A relatively high proportion of T2DM cases was documented among all diabetes patients: $24.5 \%$ within all new patients over the 16 -year period and $18.1 \%$ and $9.4 \%$ of all incident and prevalent cases, respectively, in the last year, 2016. Male preponderance was observed in the T1DM cohort and females predominated in the T2DM cohort.

The most recent International Diabetes Federation (IDF) report on diabetes in children and adolescents provides lower T1DM rates in this country. The total number of T1DM patients aged 0-19 years was estimated by the IDF as 3,166 , but in this study 3,798 were documented. The incidence rate given by the IDF is $16.7 / 100000$, in contrast to the current study, which reports 22.9/100 000 [28]. The IDF report does not give data estimates on T2DM.

This study regarding increasing T1DM incidence is in line with a previous Hungarian registry analysis of children between 1989 and 2009 [29]. However, the current study shows a slower in-

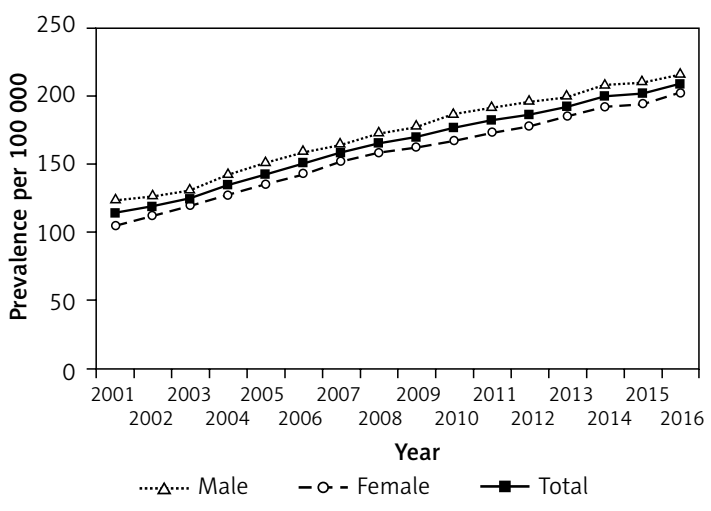

Figure 2. Prevalence of type 1 diabetes in children and adolescents aged 0-18 years in Hungary between 2001 and 2016, inclusive

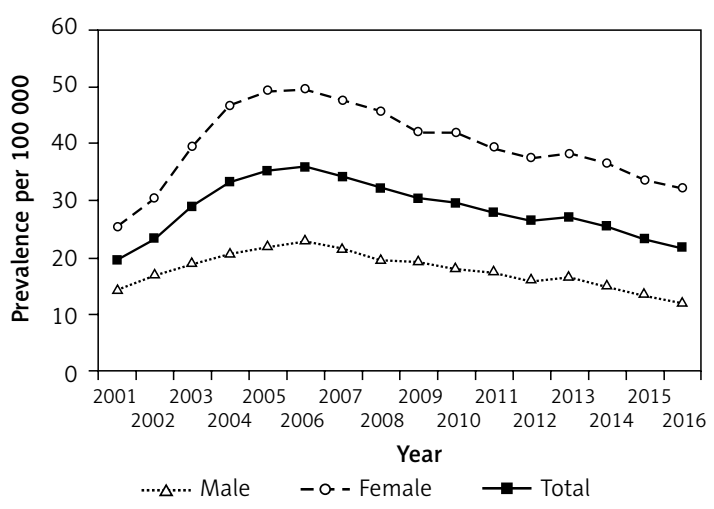

Figure 4. Prevalence of type 2 diabetes in children and adolescents aged 0-18 years in Hungary between 2001 and 2016, inclusive

crease in the incidence rate of T1DM as compared with the former study (mean annual rate of $2.5 \%$ vs. $4.4 \%$ ), although the previous study covered a substantially younger age group (0-14 years). The incidence data and its rising trend of T1DM in the current study are comparable to the findings of recent publications from the US [15, 30, 31], Australia [21] and Europe [32-35]. However, in some countries a flattened $[11,12]$ or a sinusoidal pattern [22] of T1DM incidence is observed instead of an increase. Further evaluations are necessary to assess whether a plateau will occur in Hungary over time in the future.

Few data are available on the prevalence of T1DM in youths in different countries. In a recent Dutch study the prevalence of T1DM was $143.6 / 100,000$ in children aged 14 years or younger during the years 2009-2011 [33]. Reports from the UK and US showed increasing prevalence of T1DM in children and adolescents from 108 to 198/100,000 between 1998 and 2005 and from 148 to 193/100,000 between 2001 and 2009, respectively $[16,23]$. These data are comparable to the current data from Hungary, where a change from 114 to 209/100,000 was observed between 2001 and 2016. These figures support the view 
that the growing T1DM population represents a challenge to the health care system in these countries.

Like in the current study, some recent papers showed that more males than females are affected by T1DM [15, 21, 22, 30], but some other data did not confirm this finding [16, 32, 33, 35]. In the current analysis, more male incident and prevalent T1DM cases were observed consistently in every single year and the number of all new cases among males was $25 \%$ higher than among females during the whole 16-year period, while the male population was only $5 \%$ larger than the female population. This support the previous Hungarian observation of the increasing male excess among T1DM patients in the first decade after the millennium [29].

US data describe a substantial and concordant increase in both incidence and prevalence of T2DM among youths, especially in at-risk adolescent populations [15-17, 31]. In other parts of the world, however, the picture is more subtle. In Australia, no increase in the incidence of T2DM was described during the past decade [21]. Similarly, Danish and German data also show a lack of increase in the prevalence of T2DM in children and adolescents $[19,20]$. On the other hand, rising incidence and prevalence of T2DM in youths have been observed in the UK for the past decades, especially among certain ethnic groups [22-24]. Almost all data confirm that females are more frequently affected than males $[16,18,24]$ and T2DM is a much less frequent form of diabetes than T1DM, accounting for $<10 \%$ of all diabetes cases in children and adolescents [5]. In the present study, almost 3 times more new female than male cases were registered over 16 years and no increase in the overall occurrence of T2DM was documented, which is in line with some continental European data. The finding of a decreasing male-to-female ratio of incident cases due to the decreasing number of new male cases of T2DM is noteworthy. This could be due to the sexual dimorphism in adiposity tissue function or distribution during puberty, but without anthropometric data we cannot explain this observation. Further studies are necessary to explain the reason of decreasing male-to-female ratio in new T2DM cases. However, the proportion of T2DM cases within the total diabetes population was decreasing, but surprisingly high: in 2001, T2DM accounted for $34.5 \%$ and $14.6 \%$ of the new onset and the total diabetes cases, respectively, and in 2016 it accounted for $18 \%$ and $9 \%$ of the new onset and the total diabetes cases, respectively. Furthermore, both incidence and prevalence of T2DM were higher than other European data and are more comparable to the US data. This finding could be due to the wider age band used in this study as compared to some other European assessments. Increased awareness of the health system to diagnose T2DM in this age group or earlier introduction of medication could be other reasons for the higher overall frequency of T2DM in Hungary. There are indications from some countries that a high number of patients could be misdiagnosed or undiagnosed $[20,21]$ and intervention could lead to delay in these cases. In this study, only medically treated patients were included, so the real number of T2DM patients could be even higher taking into account those on life-style intervention exclusively. Nevertheless, the finding of decreasing incidence of T2DM may indicate healthier life-style and improving health literacy, but on the other hand, decreasing awareness or resources (especially human resources) for early detection and treatment of patients could also be plausible in the recent years. Previous data from this region showed that the frequency of overweight and obese children in Hungary increased from the 1980s to the first decade of the 2000s [36]. However, according to more recent data, a stable childhood obesity rate is observed after 2010 in the country [37]. As no data are available on the obesity rate in the current study, it can be speculated that this could contribute to the decreasing tendency of T2DM in childhood. Further detailed studies are necessary to answer these questions.

There are some strengths of this study. The first is that insulin prescriptions are reliable indicators of T1DM as patients are exclusively treated with insulin since the onset. The second is that, contrary to questionnaire studies, this study does not rely on clinicians' responses, so under-reporting is assumed to be less frequent. The third is that this study consists of a population of around 2 million children and adolescents covering the whole country and providing a large database on treated T1DM and T2DM patients. The retrospective design and diagnosis based on the ICD code and prescriptions rather than clinical diagnosis are the main weaknesses of this study. Although polycystic ovary syndrome and gestational diabetes could have been excluded, some cases of monogenic diabetes, steroid induced diabetes, and cystic fibrosis related diabetes could have been misclassified. Taking into account the low occurrence of steroid induced diabetes and cystic fibrosis related diabetes, our population-based data seem to be not biased substantially by these conditions. Although no data are available on the prevalence of monogenic diabetes in Hungary, a Polish genetic screening study estimated the frequency of monogenic diabetes as $3.1-4.2 \%$ among youths with diabetes [38], highlighting the possibility of misdiagnosis of diabetes in our study. The major limitation of the study is that patients with T2DM treated with lifestyle modifications were not included, as only 
medically treated patients were identified. On the other hand, patients who were asymptomatic and left undiagnosed were not captured by this study. As a result, this study underestimated the real prevalence of the total T2DM population in the country. Another possibility is that misclassifications of T2DM patients as T1DM could have happened as no laboratory data were available to accurately classify every case retrospectively.

In conclusion, this study provides for the first time nationwide, population-based incidence and prevalence data in 2 million children and adolescents on both T1DM and T2DM parallelly in a Central-East European country over a 16-year period. T1DM is still the most common form of diabetes, and its frequency continues to rise, affecting more males. However, novel data of this study show that 1) new T2DM cases among all newly diagnosed diabetes cases in children and adolescents are much higher than expected, 2) decreasing incidence of T2DM is mainly attributable to the decreasing number of newly diagnosed male cases. Although no effective prevention is available for T1DM, screening and life-style intervention are efficient measures against the burden of T2DM. Thus, findings of this study are important for future studies, suggesting inclusion of older adolescents in analyses, and could also be useful for resource planning in the health system. Further studies are required to explain the cause of decreasing incidence of T2DM, especially among male youths.

\section{Acknowledgments}

The authors and the Hungarian Diabetes Association highly appreciate the opportunity to carry out the initiative to analyze diabetes patients' data of the Hungarian National Health Insurance Fund.

\section{Conflict of interest}

The authors declare no conflict of interest.

\section{References}

1. Katsiki N, Mikhailidis DP, Banach M. Leptin, cardiovascular diseases and type 2 diabetes mellitus. Acta Pharmacol Sin 2018; 39: 1176-88.

2. Papatheodorou K, Banach M, Bekiari E, Rizzo M, Edmonds M. Complications of Diabetes 2017. J Diabetes Res 2018; 2018: 3086167.

3. Ganjali S, Dallinga-Thie GM, Simental-Mendía LE, Banach $M$, Pirro M, Sahebkar A. HDL functionality in type 1 diabetes. Atherosclerosis 2017; 267: 99-109.

4. Mazidi M, Banach M, Kengne AP; Lipid and Blood Pressure Meta-analysis Collaboration Group. Prevalence of childhood and adolescent overweight and obesity in Asian countries: a systematic review and meta-analysis. Arch Med Sci 2018; 14: 1185-203.

5. Craig ME, Jefferies C, Dabelea D, Balde N, Seth A, Donaghue KC. Definition, epidemiology, and classification of diabetes in children and adolescents. Pediatr Diabetes 2014; 15 (Suppl. 20): 4-17.

6. Diamond T. Incidence and trends of childhood type 1 diabetes worldwide 1990-1999. Diabetic Med 2006; 23: 857-66.

7. Harron KL, McKinney PA, Feltbower RG, et al. Incidence rate trends in childhood type 1 diabetes in Yorkshire, UK 1978-2007: effects of deprivation and age at diagnosis in the South Asian and non-South Asian populations. Diabetic Med 2011; 28: 1508-13.

8. Patterson CC, Gyürüs E, Rosenbauer J, et al. Trends in childhood type 1 diabetes incidence in Europe during 1989-2008: evidence of non-uniformity over time in rates of increase. Diabetologia 2012; 55: 2142-7.

9. Newhook LA, Penney S, Fiander J, Dowden J. Recent incidence of type 1 diabetes mellitus in children 0-14 years in Newfoundland and Labrador, Canada climbs to over 45/100,000: a retrospective time trend study. BMC Res Notes 2012; 5: 628.

10. Tuomilehto J. The emerging global epidemic of type 1 diabetes. Curr Diab Rep 2013; 13: 795-804.

11. Harjutsalo V, Sund R, Knip M, Groop PH. Incidence of type 1 diabetes in Finland. JAMA 2013; 310: 427-8.

12. Berhan Y, Waernbaum I, Lind T, Möllsten A, Dahlquist G; Swedish Childhood Diabetes Study Group. Thirty years of prospective nationwide incidence of childhood type 1 diabetes: the accelerating increase by time tends to level off in Sweden. Diabetes 2011; 60: 577-81.

13. Skrivarhaug T, Stene L, Drivvoll A, Strøm H, Joner G. Incidence of type 1 diabetes in Norway among children aged 0-14 years between 1989 and 2012: has the incidence stopped rising? Results from the Norwegian Childhood Diabetes Registry. Diabetologia 2014; 57: 57-62.

14. Pinhas-Hamiel O, Dolan LM, Daniels SR, Standiford D, Khoury PR, Zeitler P. Increased incidence of non-insulin-dependent diabetes mellitus among adolescents. J Pediatr 1996; 128: 608-15.

15. Mayer-Davis EJ, Lawrence JM, Dabelea D, et al.; SEARCH for Diabetes in Youth Study. Incidence trends of type 1 and type 2 diabetes among youths, 2002-2012. N Engl J Med 2017; 376: 1419-29.

16. Dabelea D, Mayer-Davis EJ, Saydah S, et al.; SEARCH for Diabetes in Youth Study. Prevalence of type 1 and type 2 diabetes among children and adolescents from 2001 to 2009. JAMA 2014; 311: 1778-86.

17. Demmer RT, Zuk AM, Rosenbaum M, Desvarieux $M$. Prevalence of diagnosed and undiagnosed type 2 diabetes mellitus among US adolescents: results from the continuous NHANES, 1999-2010. Am J Epidemiol 2013; 178: 1106-13.

18. Rotteveel J, Belksma EJ, Renders CM, Hirasing RA, Delemarre-Van de Waal HA. Type 2 diabetes in children in the Netherlands: the need for diagnostic protocols. Eur J Endocrinol 2007; 157: 175-80.

19. Oester IMB, Kloppenborg JT, Olsen BS, Johannesen J. Type 2 diabetes mellitus in Danish children and adolescents in 2014. Pediatr Diabetes 2016; 17: 368-73.

20. Neu A, Feldhahn L, Ehehalt S, Hub R, Ranke M. Type 2 diabetes mellitus in children and adolescents is still a rare disease in Germany: a population-based assessment of the prevalence of type 2 diabetes and MODY in patients aged 0-20 years. Pediatr Diabetes 2009; 10: 468-73.

21. Tran F, Stone M, Huang C, et al. Population-based incidence of diabetes in Australian youth aged 10-18 yr: increase in type 1 diabetes but not type 2 diabetes. Pediatr Diabetes 2014; 15: 585-90. 
22. Haynes A, Bulsara MK, Bower C, Jones TW, Davis EA. Regular peaks and troughs in the Australian incidence of childhood type 1 diabetes mellitus (2000-2011). Diabetologia 2015; 58: 2513-6.

23. Hsia Y, Neubert AC, Rani F, Viner RM, Hindmarsh PC, Wong IC. An increase in the prevalence of type 1 and 2 diabetes in children and adolescents: results from prescription data from a UK general practice database. $\mathrm{Br} J$ Clin Pharmacol 2009; 67: 242-9.

24. Candler TP, Mahmoud O, Lynn RM, Majbar AA, Barrett TG, Shield JPH. Continuing rise of Type 2 diabetes incidence in children and young people in the UK. Diabetic Med 2018; 35: 737-44.

25. Śliwczyński AM, Brzozowska M, Teter Z, Marczak M, Szymański P. Regional differences in the frequency of diabetes occurrence and its treatment costs during the years 2008-2013, based on the NFZ (National Health Fund) database. Arch Med Sci 2017; 13: 256-9.

26. Niklas A, Flotyńska A, Puch-Walczak A, et al.; WOBASZ II investigators: Prevalence, awareness, treatment and control of hypertension in the adult Polish population Multi-center National Population Health Examination Surveys - WOBASZ studies. Arch Med Sci 2018; 14: 951-61.

27. Jermendy GY, Kempler P, Abonyi-Tóth ZS, Rokszin GY, Wittmann I. Changes in features of diabetes care in Hungary in the period of years 2001-2014. Orv Hetil 2016; 157: 1259-65.

28. International Diabetes Federation. IDF Diabetes Atlas, 8th edn. Brussels, Belgium: International Diabetes Federation, 2017. http://www.diabetesatlas.org

29. Gyúrûs EK, Patterson C, Soltesz G; Hungarian Childhood Diabetes Epidemiology Group. Twenty-one years of prospective incidence of childhood type 1 diabetes in Hungary - the rising trend continues (or peaks and highlands?). Pediatric Diabetes 2012; 13: 21-5.

30. Lipman TH, Levitt Katz LE, Ratcliffe SJ, et al. Increasing incidence of type 1 diabetes in youth: twenty years of the Philadelphia Pediatric Diabetes Registry. Diabetes Care 2013; 36: 1597-603.

31. Imperatore G, Boyle JP, Thompson TJ, et al.; SEARCH for Diabetes in Youth Study Group. Projections of type 1 and type 2 diabetes burden in the U.S. population aged $<20$ years through 2050: dynamic modeling of incidence, mortality, and population growth. Diabetes Care 2012; 35: 2515-20.

32. Rojnic Putarek N, Ille J, Spehar Uroic A, et al. Incidence of type 1 diabetes mellitus in 0 to 14-yr-old children in Croatia - 2004 to 2012 study. Pediatr Diabetes 2015; 16: 448-53.

33. Spaans EA, Gusdorf LM, Groenier KH, et al. The incidence of type 1 diabetes is still increasing in the Netherlands, but has stabilised in children under five (Young DUDEs-1). Acta Paediatr 2015; 104: 626-9.

34. Serban V, Brink S, Timar B, et al.; ONROCAD Study Group. An increasing incidence of type 1 diabetes mellitus in Romanian children aged 0 to 17 years. J Pediatr Endocrinol Metab 2015; 28: 293-8.

35. Fortunato F, Cappelli MG, Vece MM, et al.; Apulian Childhood-Onset Diabetes Registry Workgroup. Incidence of type 1 diabetes among children and adolescents in italy between 2009 and 2013: the role of a Regional Childhood Diabetes Registry. J Diabetes Res 2016; 2016: 7239692.

36. Bodzsár ÉB, Zsakai A. Recent trends in childhood obesity and overweight in the transition countries of Eastern and Central Europe. Ann Hum Biol 2014; 41: 263-70.

37. Kovacs VA, Bakacs M, Kaposvari CS, et al. Weight status of 7-year-old Hungarian children between 2010 and
2016 using different classifications (COSI Hungary). Obes Facts 2018; 11: 195-205.

38. Fendler W, Borowiec M, Baranowska-Jazwiecka A Szadkowska A, et al. Prevalence of monogenic diabetes amongst Polish children after a nationwide genetic screening campaign. Diabetologia 2012; 55: 2631-5. 